\title{
Review \\ Using Metabolite Data to Develop Patient Centric Specification for Amide Impurity in Vildagliptin Tablets
}

\author{
Naseem Ahmad Charoo ${ }^{1,2}$, Syeed Untoo ${ }^{3}$ and Ziyaur Rahman ${ }^{4, *}$ \\ 1 Succor Pharma Solutions, Dubai Science Park, Dubai 478861, United Arab Emirates; \\ charoo.naseem@gmail.com \\ 2 Centric Compounding LLC, Dubai Science Park, Dubai 478861, United Arab Emirates \\ 3 ICSC College of Science and Commerce, University of Kashmir, Srinagar 190001, Jammu \& Kashmir, India; \\ syeduntoo1@rediffmail.com \\ 4 Texas A\&M Health Science Center, Irma Lerma Rangel College of Pharmacy, Texas A\&M University, \\ College Station, TX 78363, USA \\ * Correspondence: rahman@tamu.edu
}

Citation: Charoo, N.A.; Untoo, S.; Rahman, Z. Using Metabolite Data to Develop Patient Centric Specification for Amide Impurity in Vildagliptin

Tablets. Sci. Pharm. 2022, 90, 1.

https://doi.org/10.3390/

scipharm 90010001

Academic Editor: William

A. Donaldson

Received: 15 November 2021

Accepted: 13 December 2021

Published: 22 December 2021

Publisher's Note: MDPI stays neutral with regard to jurisdictional claims in published maps and institutional affiliations.

Copyright: (C) 2021 by the authors. Licensee MDPI, Basel, Switzerland. This article is an open access article distributed under the terms and conditions of the Creative Commons Attribution (CC BY) license (https:// creativecommons.org/licenses/by/ $4.0 /)$.

\begin{abstract}
Many specified impurities in vildagliptin's finished product have been disclosed in the literature that are above their qualification threshold. We used the impurity B (amide impurity) as a case example to explore whether existing literature can be leveraged to determine the safe level of impurity and thereby develop a patient-centric specification (PCS) for impurities. No-observedadverse-effect level (NOAEL) was derived from rate metabolism information and converted to human equivalent dose (HED). The HED was estimated as $6.5 \mathrm{mg} /$ day. The high qualification levels are supported by repeat dose toxicity studies performed in rats, mice and dogs. Maximum theoretical amount (MTA) was correlated with the maximum observed amount (MOA) to verify whether the exposure was due to impurity and/or metabolite. MOA/MTA was found $\geq 1$ suggesting that metabolism contributed to the amount excreted in feces and therefore could be used to further justify a higher specification limit than the usual one of $\leq 0.5 \%$. Quite often the drug metabolism and degradation pathways overlap, resulting in the formation of identical constituents. Therefore, metabolism data can be leveraged for deriving safe levels of degradation impurities and develop PCS for impurities.
\end{abstract}

Keywords: patient centric specification; vildagliptin; amide impurity; repeat dose toxicity; maximum theoretical concentration; maximum observed concentration

\section{Introduction}

Three types of impurities may arise in drug substances. These are organic impurities, inorganic impurities and residual solvents. The presence of organic impurities in drug substances is attributed to the manufacturing process and/or to the degradation during shelf life. These impurities include starting materials, by products, intermediates, degradation products, reagents, ligands and catalysts. They can be identified or unidentified and volatile or non-volatile. On the other hand, inorganic impurities are generally known and identified, and produced directly from the manufacturing process. Examples of these impurities are reagents, ligands, catalysts, heavy metals or other residual metals, inorganic salts and other materials such as filter aids, charcoal, etc. Organic or inorganic solvents which are required during the synthesis of a new drug substance may be present in the drug substance as residual impurities. Based on their potential risk to human health, they are divided into three categories, i.e., Class I (solvents to be avoided), II (solvents to be limited) and III (solvents with low toxicity potential). The toxicity of these solvents is generally known, and appropriate controls are used in the specifications. There are other extraneous contaminants that may result from non-compliance to good manufacturing practice (GMP) and contaminate drug substance and product. Apart from these impurities, 
polymorphic forms and enantiomeric impurities also impact the safety and efficacy of the drug substance, hence these should be controlled in the specifications [1,2].

The ideal scenario would be to produce a drug substance with $100 \%$ purity (without any impurity). However, such a process would have a deleterious impact on the environment, physical properties (particle size, density, surface area etc.), cost, waste generation, etc. Therefore, trade-offs between purity and the presence of impurities in the drug substance or drug product is inevitable. Consequently, the impurities are controlled in drug substance and drug product specifications. Guidance documents recommend the degradation products present at a level greater than the reporting threshold be summed and reported as total degradation products [1-3]. The degradation products should be identified if their level exceeds the identification threshold provided in the guidance documents (Table 1). Likewise, degradation products present at levels greater than the qualification threshold must be qualified. The qualification of impurities is defined as "the process of acquiring and evaluating data that establishes the biological safety of an individual impurity or a given impurity profile at the level(s) specified." If nonclinical or clinical safety studies don't support the impurity levels above the qualification threshold, then new safety studies must be performed to qualify such impurities [1,2].

Table 1. Thresholds for impurities (degradation products) in new drug products.

\begin{tabular}{|c|c|}
\hline $\begin{array}{l}\text { Maximum Daily Dose } \\
\text { (Amount of Drug Substance } \\
\text { Administered per Day) }\end{array}$ & $\begin{array}{c}\text { Threshold } \\
\text { (Expressed Either as a Percentage of the Drug } \\
\text { Substance or as Total Daily Intake of the } \\
\text { Degradation Product) }\end{array}$ \\
\hline \multicolumn{2}{|c|}{ Reporting thresholds } \\
\hline$\leq 1 \mathrm{~g}$ & $0.1 \%$ \\
\hline$>1 \mathrm{~g}$ & $0.05 \%$ \\
\hline \multicolumn{2}{|c|}{ Identification Thresholds } \\
\hline$<1 \mathrm{mg}$ & $1.0 \%$ or $5 \mu \mathrm{g}$ TDI, whichever is lower \\
\hline $1 \mathrm{mg}-10 \mathrm{mg}$ & $0.5 \%$ or $20 \mu \mathrm{g}$ TDI, whichever is lower \\
\hline$>10 \mathrm{mg}-2 \mathrm{~g}$ & $0.2 \%$ or $2 \mathrm{mg}$ TDI, whichever is lower \\
\hline$>2 \mathrm{~g}$ & $0.10 \%$ \\
\hline \multicolumn{2}{|c|}{ Qualification Thresholds } \\
\hline$<10 \mathrm{mg}$ & $1.0 \%$ or $50 \mu \mathrm{g}$ TDI, whichever is lower \\
\hline $10 \mathrm{mg}-100 \mathrm{mg}$ & $0.5 \%$ or $200 \mu \mathrm{g}$ TDI, whichever is lower \\
\hline$>100 \mathrm{mg}-2 \mathrm{~g}$ & $0.2 \%$ or $3 \mathrm{mg}$ TDI, whichever is lower \\
\hline$>2 \mathrm{~g}$ & $0.15 \%$ \\
\hline
\end{tabular}

The FDA manual of policies and procedures (MAPP) 5017.2 published in 2018 placed clinical relevance at the centre stage while establishing acceptance criteria for impurities in drug substance and drug product for non-mutagenic impurities in new drug applications (NDAs), abbreviated new drug applications (ANDAs), and biologics license applications (BLAs) [4]. The MAPP envisioned two scenarios for establishing acceptance criteria for impurities with acceptance criteria $\leq \mathrm{ICH}$ Q3A(R2) or Q3B(R2) qualification threshold [1,2]:

1. Acceptable limit for a specified impurity can be set at the qualification threshold provided no toxicological, immunological, or clinical concerns exist at this level.

2. Potent and toxic impurities having immunological, pharmacological, or clinical concerns: Acceptance criteria based solely on ICH Q3A(R2) and Q3B(R2) qualification threshold are not enough and need further justification.

While MAPP lays emphasis on deriving acceptance criteria for specified impurities based on their clinical impact, it also notes that the manufacturing process consistency should be monitored during the production run, especially for impurities exhibiting a high degree of uncertainty [4]. For instance, impurities which degrade further to generate other impurities may require a stringent manufacturing process control. Notwithstanding this, specifications established based on process experience alone don't consider the impact of process boundaries on the product's safety and efficacy in patients. Patient centric specifi- 
cations [(PCS) (also known as clinically relevant specifications)] take into consideration the clinical impact of variations in the critical quality attributes (CQAs) and process parameters, thereby assuring a consistent safety and efficacy profile [5,6]. PCS accept or reject batches based on their performance in an indicated patient population. In fact, it resonates well with the central theme of ICHQ6A, i.e., the specifications should focus on safety and efficacy and not on batch data [7]. MAPP 5017.2 defines clinically relevant acceptance criteria as a set of acceptance ranges to which an impurity should conform in order for the product to be safe and effective when used as labelled [4]. The easier approach is to evaluate identified impurities by using the existing literature and database. If prior knowledge is inadequate to support impurity levels in a product, then structure-based (in silico) assessment for mutagenicity is carried out using two different computational methods: rule based and statistical based, followed by assessment of the computational results by an expert. ICH M7 categorises impurities into five classes based on their mutagenic and carcinogenic potential. For mutagenic impurities (Class I, 2 and 3), controls recommended are either compound specific (Class I) or threshold of toxicological concern (TTC) based (Class 2 and 3). For non-mutagenic impurities (Class 4 and 5), controls recommended in ICH Q3A and Q3 B can be used [8].

Many public assessment reports leading to the marketing authorisation of vildagliptin and vildagliptin/metformin tablets report the level of various impurities above qualification threshold $[9,10]$. Literature data also suggested that these impurities are non-mutagenic impurities. Using the impurity B (amide impurity) as a case example, we explore how existing literature can be leveraged to determine the safe level of impurity and thereby, develop a PCS for impurities.

\section{Methods}

The pharmacokinetics and pharmacodynamics property of vildagliptin was extracted from literature data available online in the public domain using the following keywords: vildagliptin, metabolism, pharmacokinetics, pharmacodynamics, mechanism of action, dose, impurity B, amide impurity and degradation impurities. The data were systematically examined.

The human equivalent dose $(H E D)$ was calculated by using the following formula [11]:

$$
H E D=N O A E L \div\left[\frac{K_{\text {mhuman }}}{k_{\text {mrat }}}\right]
$$

A value of 6.2 was used for $K_{\text {mhuman }} / K_{\text {mrat }}[11]$.

The maximum theoretical concentration (MTC) and the maximum observed concentration (MOC) were estimated by using a method proposed by Weidolf and co-workers [3]. Chemical structures were drawn using ACD/ChemSketch (ACD/Labs version 2020).

\section{Results and Discussion}

\subsection{Vildagliptin Metabolism and Degradation}

Vildagliptin is (S)-1-[2-(3-Hydroxyadamantan-1-ylamino)acetyl]pyrrolidine-2-carbonitrile. It is a selective and reversible inhibitor of enzyme dipeptidyl peptidase 4 (DPP-4). This enzyme inactivates the incretin hormones, glucagon-like peptide-1 (GLP-1), and glucosedependent insulinotropic polypeptide (GIP) which significantly contribute to the maintenance of glucose homeostasis. Consequently, vildagliptin increases the fasting and postprandial endogenous levels of GLP-1 and GIP and consequently enhances the sensitivity of beta cells to glucose. Vildagliptin has also been found to enhance the sensitivity of alpha cells to glucose resulting in more glucose appropriate glucagon secretion. It is indicated for treating type 2 diabetes mellitus in adults either as monotherapy or as dual therapy in combination with metformin, sulphonylurea and a thiazolidinedione or as a triple therapy with a sulphonylurea and metformin or in combination with insulin with or without metformin [9-12]. The recommended daily dose of vildagliptin in adults is $100 \mathrm{mg}$, which can be administered in two divided doses of $50 \mathrm{mg}$ each; one dose of $50 \mathrm{mg}$ in the morning and one dose of $50 \mathrm{mg}$ in the evening. When vildagliptin is used in combination with suphonylurea, the recommended dose of vildagliptin is $50 \mathrm{mg}$ once daily adminis- 
tered in the morning. Vildagliptin/metformin can be administered as $50 \mathrm{mg} / 850 \mathrm{mg}$ or $50 \mathrm{mg} / 1000 \mathrm{mg}$ twice daily (12).

Vildagliptin is rapidly absorbed in rats and dogs and exhibits moderate to high bioavailability (45-100\%), suggesting low first pass metabolism [13-15]. In humans also, absorption is rapid with absolute bioavailability 85\% [12]. A major portion is excreted unchanged in rats and dogs. All the metabolites reported in human studies have also been detected in rats and dogs. The main metabolism pathway involves hydrolysis at the cyano and amide bonds to form M20.7 and M15.3, respectively. Hydrolysis of the cyano group appears to be a 2-step reaction leading to the formation of M18.6 and subsequently a free acid (M20.7). Conversion might be rapid, leading to low concentrations of M18.6. As these metabolites were not formed in liver microsomes, the role of cytochrome p450s in vildagliptin metabolism is ruled out [15]. However, M20.7 and M15.3 together with M20.2, which is generated through the glucuronidation pathway, suggests metabolism via hydrolysis and glucuronidation routes in liver (Table 2). M20.7 is the main metabolite both in the rat (54\%) and $\operatorname{dog}(33 \%)$. The metabolites that were produced only in rats and/or dogs included [13-15]:

- M14.9 (hydroxylation at the adamantyl ring)

- M16.7 (hydroxylation at the pyrrolidine ring)

- M17.4 (hydroxylation at the pyrrolidine ring of M20.7)

- M18.6 (amide metabolite resulting from hydrolysis of the cyano moiety)

- M14.2 (amide metabolite)

- $\quad$ M17.7 (a monohydroxy-acid metabolite resulting from the ring opening of the pyrrolidine ring)

Table 2. Metabolites of vildagliptin in rats, dogs and humans.

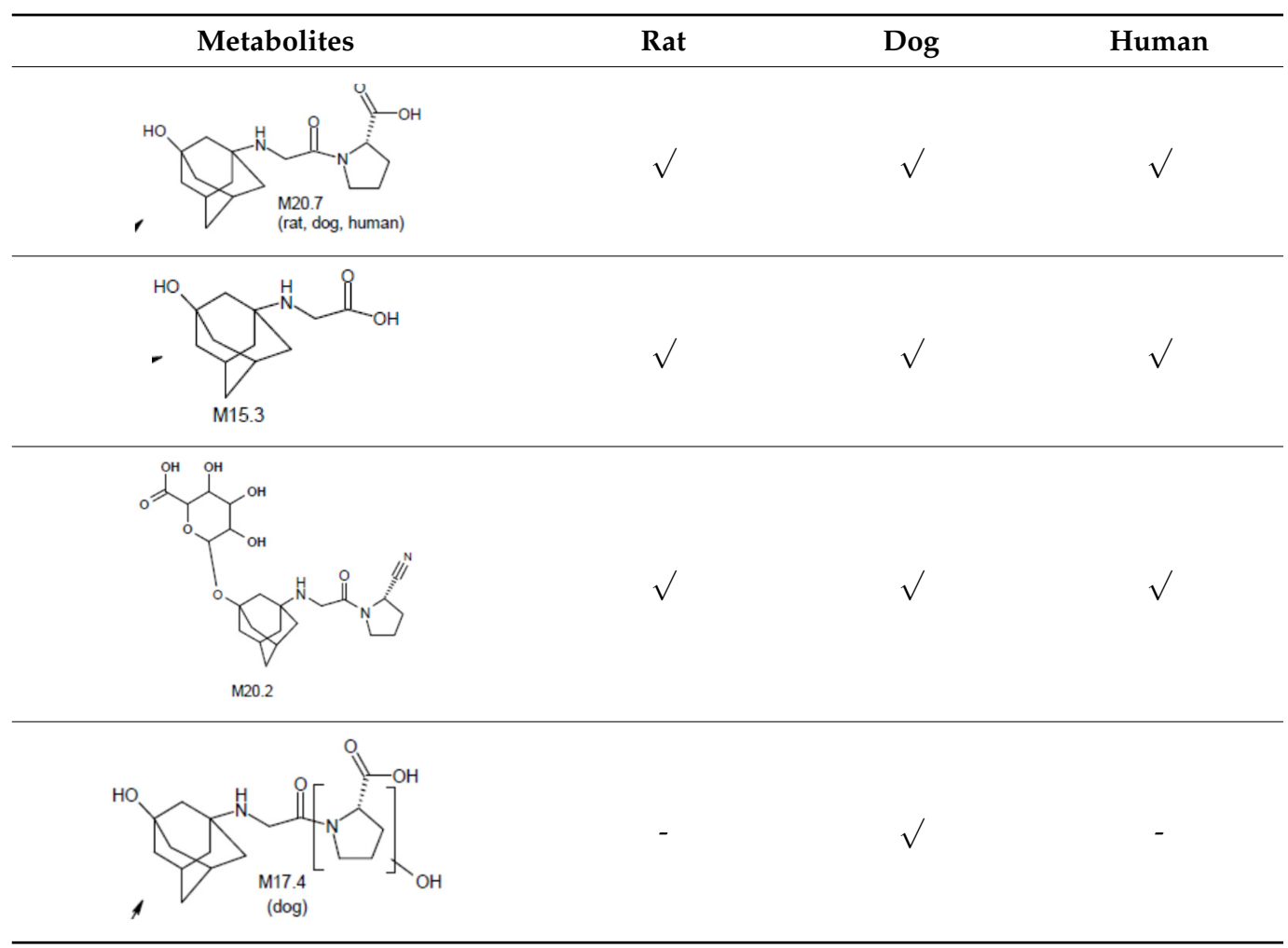


Table 2. Cont.

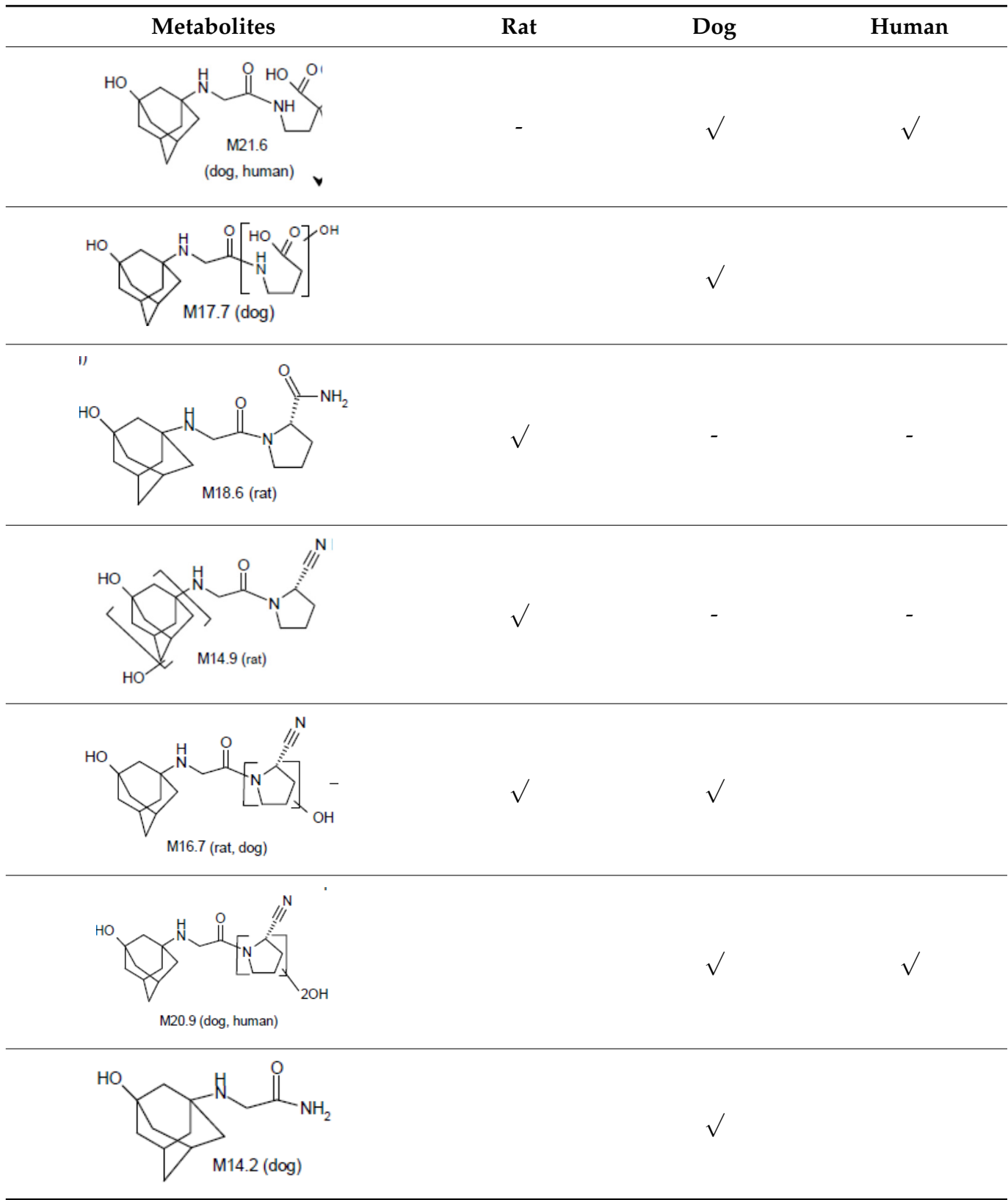

In humans, approximately $69 \%$ of the dose is metabolised, the major metabolite being [(LAY 151 (M20.7)] a cyano moiety (57\% of the dose). The hydrolysis of vildagliptin is believed to take place in the kidney, resulting in the formation of LAY 151. Like in the case of rats and dogs, it is not metabolised by CYP450 and hence its metabolic clearance is not influenced by CYP 450 inhibitors and/or inducers, thus making it less susceptible to pharmacokinetic interactions with P 450 inducers and inhibitors. Other minor metabolites observed in humans result from amide bond hydrolysis (M15.3), glucuronidation (M20.2), or oxidation on the pyrrolidine moiety of vildagliptin (M20.9 and M21.6) (Table 2) $[12,14,15]$.

Various process and degradation impurities of vildagliptin have been reported $[16,17]$. These include:

- $\quad\left(2 \mathrm{~S}, 2 \mathrm{~S}^{\prime}\right)-1,1^{\prime}[[3$-hydroxytricyclo[3.3.1.1.3,7]dec-1-yl)imino]bis(1-oxo2,1,-ethanediyl]bis (2-pyrrolidinecarbonitrile) impurity (Dimer impurity) of formula (VI);

- aminoadamantane-3-ol impurity of formula (IV);

- Adamantane-1,3-diol impurity (Di-hydroxyl impurity) of formula (VII) 
- deshydroxy impurity of formula (VIII)

- $\quad$ amide impurity of formula (IX)

- $\quad$ impurity E ((2S)-1-[2-[(3-hydroxyadamantan-1-yl)imino]acetyl]pyrrolidine-2-carbonitrile)

- $\quad$ impurity F ((8aS)-3-hydroxy-octahydropyrrolo[1,2-a]piperazine-1,4-dione)

\subsection{Estimating Safe Level of Amide Impurity (Impurity B)}

Many specified impurities in vildagliptin's finished product have been disclosed in the literature that are above their qualification threshold. One of the specified impurities is M18.6 (impurity B or amide impurity). Arar and co-workers reported that impurity B is formed under oxidative and basic stress conditions (Figure 1) [18]. The hydrolysis of vildagliptin to impurity $B$ also occurs at a very high rate under higher levels of humidity and high temperature [19]. While impurity $B$ is not reported to form during metabolism in humans, He et al. found that the vildagliptin amide derivative (M18.6 or impurity B) was formed as a minor metabolite in rats after oral administration of vildagliptin $100 \mathrm{mg} / \mathrm{kg}$ body weight. M18.6 was excreted in faeces and formed $1.5 \%$ of the total administered dose. Considering bioavailability of $45 \%$ in rats, an exposure of around $0.135 \mathrm{mg}$ per rat $(0.675 \mathrm{mg} / \mathrm{kg})$ is expected [13].

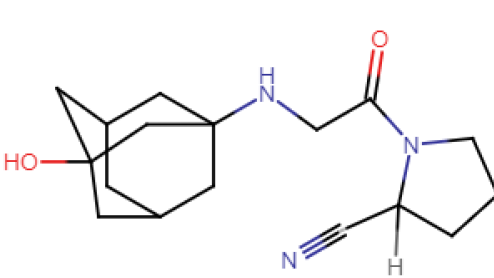

Vildagliptin

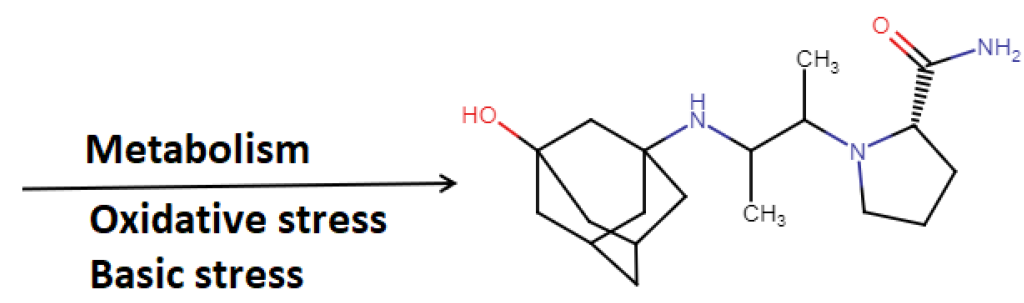

Amide impurity (M18.6)

Figure 1. Metabolism/degradation of vildagliptin to amide impurity/M18.6 metabolite.

Repeat dose toxicity studies were performed in rats and dogs for up to 26 weeks and 52 weeks, respectively. In these studies, drug impurities observed at higher levels than the qualification threshold were tested by spiking vildagliptin preparation with the impurities at levels of 2-3\%. No toxicity potential was observed, and no safety concerns of clinical relevance were apparent [20]. Further, it did not show any genotoxic, carcinogenic or reproductive toxicity potential. Moreover, it didn't show any local irritation potential. Thus, the animal toxicity studies didn't indicate any toxicity potential due to metabolites. With $0.675 \mathrm{mg} / \mathrm{kg}$ exposure after a single dose, it can be safely assumed that exposure to M18.6 over 26 weeks would be much higher considering it is formed as a metabolite in rats. In a different study, vildagliptin exhibited an excellent safety profile in a rodent toxicity study performed at oral doses up to $900 \mathrm{mg} / \mathrm{kg}$ (200 times more than human exposure) for 104 weeks in rats and in mice at oral doses up to $1000 \mathrm{mg} / \mathrm{kg}$ (up to 240 times the human exposure at the maximum recommended dose) [21]. The exposure to M18.6 would have been even higher.

Assuming no-observed-adverse-effect-level $(\mathrm{NOAEL})=0.675 \mathrm{mg} / \mathrm{kg} /$ day; the HED for a $60 \mathrm{~kg}$ individual and using a factor of 6.2 [(for converting animal dose (in $\mathrm{mg} / \mathrm{kg}$ ) to HED (in $\mathrm{mg} / \mathrm{kg}$ )] would be $6.5 \mathrm{mg}$. Based on the above data, impurity B at levels $\leq 6.5 \mathrm{mg}$ / day can be considered qualified.

The above estimated safe level of impurity B is further supported by a four week repeat dose toxicity study that was performed in rats to qualify a test product which contained $2.8 \%, 2.0 \%$ and $1.9 \% \mathrm{w} / \mathrm{w}$ of vildagliptin impurities. Although the impurity names have not been disclosed, it is understood that one of the impurities is impurity B, the commonly seen degradation product of vildagliptin. The test product was administered at a dose corresponding to $900 \mathrm{mg} / \mathrm{kg}$ /day of vildagliptin. No difference in toxicity was 
observed between the test product and unspiked vildagliptin, suggesting a substantial safety margin at the maximum human dose of vildagliptin (animal/human =130/600). Genotoxic studies were negative in a bacterial reverse mutation assay at vildagliptin concentrations $\leq 5 \mathrm{mg}$ /plate, and were negative in the chromosome aberration assay at vildagliptin concentrations $\leq 3 \mathrm{mg} / \mathrm{mL}[9,10]$.

\section{The Maximum Theoretical Concentration (MTC) versus Maximum Observed Concentration (MOC)}

Both drug metabolism and degradation (during processing and / or storage) generally undergo similar chemical transformation resulting in the formation of identical constituents. Consequently, many impurities generated during degradation are also metabolites. ICH guidance documents state that, "Degradation products that are also significant metabolites present in animal and/or human studies are generally considered qualified" [1,2]. Therefore, if biological safety is demonstrated by proving that in vivo exposure to the metabolite is equal to or greater than the maximum theoretical exposure due to exposure to impurity (from the drug substance's degradation), further animal testing may not be required. In fact, FDA MAPP also observes that "acceptance criterion for individual impurities that are also significant human metabolites should be considered separately" [4]. The major challenge, however, remains that the guidance documents don't specify how the comparison between impurity and metabolite should be made to verify whether the exposure is due to impurity or metabolite or both. However, guidance documents observe that "Nonclinical characterization of a human metabolite(s) is only warranted when that metabolite(s) is observed at exposures greater than $10 \%$ of total drug-related exposure (DRE) and at significantly greater levels in humans than the maximum exposure seen in the toxicity studies". The impurities which are also significant metabolites $(>10 \%)$ can be assessed in clinical and non-clinical studies (MIST: metabolites in safety testing). The known metabolites can be used to establish whether one or more impurities are present as metabolites and have been exposed to animals or man. Notwithstanding this, as observed by the FDA, MAPP metabolites at $<10 \%$ of total DRE found in animals or humans would still need to be qualified to prove their biological safety [4].

An elementary comparison of an impurity with a detected metabolite in humans or animals is not adequate to qualify an impurity because the detected compound could be a metabolite or even an impurity. Therefore, it is imperative to establish that an exposure is equal to or greater than the level that might result from the exposure to the actual impurity following administration of the drug substance or drug product. To address this, Weidolf and co-workers proposed a quantitative methodology for comparing circulatory impurity and/or metabolite levels to the impurity levels to be qualified in the drug substance or drug product [3]. The authors proposed comparing maximum theoretical concentration (MTC) versus maximum observed concentration (MOC) using non-clinical pharmacokinetics and toxicity studies and clinical study data to establish whether the source of the exposure can indeed be attributed to the impurity content alone or whether metabolism contributes to its formation. For estimating MTC, the authors considered a worst-case scenario by assuming the complete bioavailability, with no protein binding, no distribution into blood cells or other tissues and no elimination (metabolic or non-metabolic). Under these conditions, the observed plasma concentration is expected to be the same as the concentration in the extracellular fluid (ECF) and will also hold true regardless the substance is impurity or formed metabolically. The calculated MTC is then compared to the MOC followed by evaluation if the exposure can be attributed to impurity alone or to metabolism. Intra species comparison of MTC versus MOC would verify the contribution of the metabolite to the impurity/metabolite levels (MOC > MTC). On the other hand, inter species comparisons would establish if exposure to the impurity/metabolite is higher in animals than in humans. The authors also suggested that MOC animal data should be determined at the NOAEL of the drug substance whose impurity is to be evaluated. The dose used in the human MTC calculation should be the maximum recommended human dose. 
The reliability of this approach in supporting qualification of impurity is governed by the fact that the plasma levels of the impurity should be at least equivalent to the maximum theoretical impurity exposure. Urinary excretion data, if available, would provide an estimate of absolute exposure and would not depend on pharmacokinetics assumptions.

The authors envisaged two scenarios:

1. MOC < MTC: Circulating level is below the to be qualified level. In this case further information (such as in vitro metabolism studies, in vivo PK studies, assessment of coverage across species or urinary excretion) would be required to support the qualification of the impurity. If these studies fail to provide evidence of metabolite formation or interspecies coverage, then GLP toxicity impurity qualification studies would be required.

2. MOC $\geq$ MTC: Circulatory level is above or equal to the level to be qualified, the circulating level can be used to qualify an impurity.

The concept can also be used for renally excreted metabolites. The term maximum theoretical amount (MTA analogous to MTC) was used for renally excreted metabolites. Applying the latter approach to vildagliptin impurity B (Tables 2 and 3), the amount of impurity B excreted in faeces is equal to the theoretically expected value if the impurity is $100 \%$ bioavailable and $100 \%$ excreted. On the other hand, if bioavailability is $45 \%$ (reported in literature for rats), then the amount of impurity B excreted in faeces is 2.2 times greater than the theoretically expected value. This suggests that metabolism contributes to the amount excreted in faeces and could be used to justify a higher specification limit than the usual limit (which is $\leq 0.5 \%$ ).

Table 3. Observed metabolite and theoretical impurity amounts of impurity B formed from vildagliptin and excreted in faeces following oral administration in rats.

\begin{tabular}{ccc}
\hline \multirow{2}{*}{ Parameter } & \multicolumn{2}{c}{ Values } \\
\cline { 2 - 3 } & Scenario 1 & Scenario II \\
\hline Maximum Dose $(\mathrm{mg})$ & 20 & 20 \\
Average dose of impurity $(\mathrm{mg})$ & 0.135 & 0.3 \\
Average dose of impurity $(\mu \mathrm{g})$ & $135^{*}$ & $300^{\#}$ \\
\% of dose excreted in urine & 1.5 & 1.5 \\
MTA of impurity $(\mu \mathrm{g})$ & 135 & 300 \\
MOA excreted in urine $(\mu \mathrm{g})$ & 300 & 300 \\
MOA/MTA & 2.2 & 1.0 \\
\hline
\end{tabular}

* Considering bioavailability of 0.45 ; \# Considering bioavailability of 1.0 .

\section{Conclusions}

Quite often the drug metabolism and degradation pathways overlap, resulting in formation of identical constituents. Therefore, metabolism data can be leveraged for deriving safe levels of degradation impurities and develop PCS for impurities. By way of example, we have used the rat metabolism information to derive the NOAEL for vildagliptin amide impurity and converted it into HED. Furthermore, MTA was correlated with MOA to verify if the metabolism contributes to the in vivo exposure to the impurity. PCS was determined, taking into consideration the patient requirements for safety and efficacy (clinical relevance). Additionally, prior knowledge was leveraged to enhance the product understanding and link product quality to clinical performance. The approach offers flexibility in setting specifications for CQAs that are clinically acceptable. These in turn would ensure the supply chain continuity and cost control.

Author Contributions: Conceptualization and methodology: N.A.C., Z.R. and S.U. Data Analysis: N.A.C., Writing-review and editing, N.A.C., Z.R. and S.U. All authors have read and agreed to the published version of the manuscript.

Funding: This research received no funding. 
Institutional Review Board Statement: Not applicable.

Informed Consent Statement: Not applicable.

Data Availability Statement: Not applicable.

Conflicts of Interest: The authors declare no conflict of interest.

\section{References}

1. International Conference on Harmonization (ICH) —Impurities in New Drug Substances Q3A(R2). 2006. Available online: https:/ / database.ich.org/sites/default/files/Q3A\%28R2\%29\%20Guideline.pdf (accessed on 10 November 2021).

2. International Conference on Harmonization (ICH)_Impurities in New Drug Products Q3B(R2). 2006. Available online: https: / / database.ich.org/sites/default/ files/Q3B\%28R2\%29\%20Guideline.pdf (accessed on 10 November 2021).

3. Weidolf, L.; Andersson, T.; Bercu, J.; Brink, A.; Glowienke, S.; Harvey, J.; Hayes, M.; Jacques, P.; Lu, C.; Manevski, N.; et al. Qualification of impurities based on metabolite data. Reg. Tox. Pharmacol. 2020, 110, 104524. [CrossRef] [PubMed]

4. $\quad$ FDA MAPP 5017.2 Rev. 1. Manual of Policy and Procedures, Guidance for Assessors; “Establishing Impurity Acceptance Criteria as Part of Specifications for NDAs, ANDAs, and BLAs Based on Clinical Relevance". 2020. Available online: https: / / www.fda.gov/media/124859/download (accessed on 10 November 2021).

5. Sharp, S.S. Establishing Clinically Relevant Drug Product Specifications: FDA Perspectives. Presentation at AAPS Annual Meeting. 2012. Available online: https://www.fda.gov/media/85355/download (accessed on 10 November 2021).

6. Bercu, J.; Berlam, S.C.; Berridge, J.; Cherney, B.; Cowley, D.; Laughton, H.W.; McLoughlin, D.; McMahon, M.; Moore, C.M.V.; Murti, C.; et al. Establishing patient centric specifications for drug substance and drug product impurities. J. Pharm. Inn. 2019, 14, 76-89. [CrossRef]

7. International Conference on Harmonization (ICH)—Specifications: Test Procedures and Acceptance Criteria for New Drug Substances and New Drug Products: Chemical Substances, Q6A. 1999. Available online: https://database.ich.org/sites/default/ files/Q6A\%20Guideline.pdf (accessed on 10 November 2021).

8. International Conference on Harmonization (ICH)-Assessment and Control of DNA Reactive (Mutagenic) Impurities in Pharmaceuticals to Limit Potential Carcinogenic Risk M7(R1). 2017. Available online: https:/ /www.ema.europa.eu/en/documents / scientific-guideline/ich-guideline-m7r1-assessment-control-dna-reactive-mutagenic-impurities-pharmaceuticals-limit_en.pdf (accessed on 10 November 2021).

9. Australian Public Assessment Report for Vildagliptin. Proprietary Product Name: Galvus, Xiliarx. Sponsor: Novartis Pharmaceuticals Australia Pty Ltd. 2010. Available online: https:/ /www.tga.gov.au/sites/default/files/auspar-galvus.pdf (accessed on 12 July 2020).

10. Australian Public Assessment Report for Vildagliptin/Metformin Hydrochloride. Proprietary Product Name: Galvumet/Sobrea. Sponsor: Novartis Pharmaceuticals Australia Pty Ltd. 2011. Available online: https://www.tga.gov.au/auspar/ausparvildagliptin-metformin-hydrochloride-0 (accessed on 10 November 2021).

11. FDA. Estimating the Maximum Safe Starting Dose in Initial Clinical Trials for Therapeutics in Adult Healthy Volunteers. 2005. Available online: https:/ / www.fda.gov/media/72309/download (accessed on 10 November 2021).

12. Summary of Product Characteristics (SmPC). Galvus $50 \mathrm{mg}$ Tablets. 2018. Available online: https://www.medicines.org.uk/ EMC/medicine/20734/SPC/Galvus+50+mg+Tablets/ (accessed on 10 November 2021).

13. He, H.; Tran, P.; Yin, H.; Smith, H.; Flood, D.; Kramp, R.; Filipeck, R.; Fischer, V.; Howard, D. Disposition of Vildagliptin, a Novel Dipeptidyl Peptidase 4 Inhibitor, in Rats and Dogs. Drug Metabol. Disp. 2009, 37, 545-554. [CrossRef] [PubMed]

14. He, H.; Tran, P.; Yin, H.; Smith, H.; Batard, Y.; Wang, L.; Finolf, H.; Gu, H.; Manglod, J.B.; Fischer, V.; et al. Absorption, metabolism, and excretion of [14C]vildagliptin, a novel dipeptidyl peptidase 4 inhibitor, in humans. Drug Metab. Dispos. 2009, 37, 536-544. [CrossRef] [PubMed]

15. He, L.H.; Serra, D.; Wang, Y.; Campestrini, J.; Riviere, G.J.; Deacon, C.F.; Schwartz, H.S.; Nielsen, J.C.; Saylan, M.L. Pharmacokintics and Pharmacodynamics of vildagliptin in patients with type 2 diabetes mellitus. Clin. Pharmacokin. 2007, 46, 577-788. [CrossRef] [PubMed]

16. Kumar, N.; Devineni, S.R.; Singh, G.; Kadirappaa, A.; Dubey, S.K.; Kumar, P. Identification, isolation and characterization of potential process-related impurity and its degradation product in vildagliptin. J. Pharm. Biomed. Anal. 2015, 119, 114-121. [CrossRef] [PubMed]

17. Thippannachar, M.V.; Sudhir, N.; Vrajlal, P.; Subhash, I.S.; Bhagirath, B.M.; Rajesh, T.; Chaturlal, M.A. A Process for the Preparation of Vildagliptin and Its Intermediate Thereof. WO Patent 2013179300 A3, 28 August 2014.

18. Arar, S.; Al-Qudah, E.; Alzweiri, M.; Sweidan, K. New forced degradation products of vildagliptin: Identification and structural elucidation using LC-MS, with proposed formation mechanisms. J. Liq. Chromat. Rel. Technol. 2020, 43, 633-644. [CrossRef]

19. Tianjiao, H.; Guanghui, L.; Hongguo, L.; Yingtao, T.; Ning, X.; Jing, Z.; Tianjiao, H.G. Vildagliptin Tablet and Preparation Method Thereof. China Patent CN105193752B, 30 March 2018. 
20. EMEA-Vildagliptin Scientific Discussion. 2007. Available online: https://www.ema.europa.eu/en/documents/scientificdiscussion/eucreas-epar-scientific-discussion_en.pdf (accessed on 10 November 2021).

21. Busch, S.J.; Hoffmann, P.; Sahota, P.; Johnson, R.; Kothny, W.; Meyer, F.; Foley, J.E. Studies in rodents with the dipeptidyl peptidase4 inhibitor vildagliptin to evaluate possible drug-induced pancreatic histological changes that are predictive of pancreatitis and cancer development in man. Diabetes Obes. Metab. 2013, 15, 72-76. [CrossRef] [PubMed] 